\title{
HBB NM_000518.4:C.20A>T
}

National Cancer Institute

\section{Source}

National Cancer Institute. HBB NM 000518.4:C.20A>T. NCI Thesaurus. Code C156902.

A nucleotide substitution at position 20 of the coding sequence of the HBB gene where adenine has been mutated to thymine. 